\title{
Computational Modeling and Experimental Evaluation of the Effects of Electrode Geometry and Deposition Target on Electrostatic Spraying Processes
}

\author{
Manoj K. Patel \\ ${ }^{1}$ Academy of Scientific \\ and Innovative \\ Research, AcSIR-CSIO, \\ Chandigarh, 160 030, IN \\ ${ }^{2}$ CSIR-Central Scientific \\ Instruments \\ Organisation, \\ Chandigarh, 160 030, IN
}

\author{
Tanveer Sharma \\ Giani Zail Singh Punjab \\ Technical University, \\ Bathinda, 151 001, IN
}

\author{
Manoj K. Nayak \\ ${ }^{1}$ Academy of Scientific \\ and Innovative \\ Research, AcSIR-CSIO, \\ Chandigarh, 160 030, IN \\ ${ }^{2}$ CSIR-Central Scientific \\ Instruments \\ Organisation, \\ Chandigarh, 160 030, IN
}

\author{
C. Ghanshyam \\ ${ }^{1}$ Academy of Scientific \\ and Innovative \\ Research, AcSIR-CSIO, \\ Chandigarh, 160 030, IN \\ ${ }^{2}$ CSIR-Central Scientific \\ Instruments \\ Organisation, \\ Chandigarh, 160 030, IN
}

\begin{abstract}
Electrostatic force field application is one of the most promising methods for spraying protective liquid sprays onto the biological surfaces of crops, orchards, vineyards and trees, because electrostatic space charge and image deposition forces enhance the uniformity of spray on the target surface and increases the transfer efficiency, mass transfer and adhesion. The study has been carried out to know the effects of electrode geometry, deposition target and spray cloud through computational modeling using COMSOL Multiphysics. In this work, four different shaped targets, four different geometry of electrodes and four stages of spray cloud are analyzed to know the effects of resultant electric field to charge the conductive liquid sprays. Comparative study of electric field among all the four shaped targets resulted that elliptical target has the maximum resultant electric field followed by conical, spherical and cylindrical target respectively. Similarly in case of electrode geometry, electric field of square electrode with circular cross-section found to be optimum followed by circular electrode with circular cross-section, circular electrode with square cross section and square electrode with square cross section respectively. The experimental results are in good agreement with computational modeling of charging the liquid sprays.
\end{abstract}

\section{General Terms}

Agriculture processes, Computational modeling, Electrostatic spraying, COMSOL Multiphysics

\section{Keywords}

Aerodynamics, Deposition characteristics, Electrodynamics, Hydrodynamics, Induction charging, Spray cloud

\section{INTRODUCTION}

The electrostatic sprayer is a system that electrically charges the finely divided particulate matter inductively [1-5]. To use the technology efficiently, it is very important to optimize the parameters of the spray nozzle. An attraction force of a charged particle to a plant consists of two reasons i.e. due to the action of the electrostatic field of the particle itself and the electric field forces of the spray tip and the cloud upon the electric field of the drop [6-9]. If electric fields are directed to the plant, drops will be projected onto its surface. Research approaches to agricultural spray charging which is based upon several distinct principles [10-14]. Maski and Durairaj showed that a major portion of the electrostatic pesticide spraying has been in the development of reliable means for droplet charging [15]. Motion of the charged particle can be easily controlled by the electric force, which depends on the charging level. Therefore, it is desirable to charge the finely divided particulate matter as high as possible and the charged droplet must be acted upon by an electric field. This electric field may be self-generated or externally applied. The technique uses a combination of charge transformation and surface tension variation by interaction phenomenon of compressed air supply, liquid flow and electrostatic field to generate uniformly charged spray droplets. Effective surface tension variation by electrostatic force applied to fluid jet and air jet, imparted perturbation to fluid jet results in charged droplet formation [16, 17]. The performance parameters of electrostatic spray nozzle i.e. charge-to-mass ratio is a result of complex interactions of input parameters i.e. compressed air supply, liquid flow and electrostatic field within the designed nozzle.

Despite of available literature, it is obvious that very little research work has been carried out on the optimization of electrostatic nozzle parameters using COMSOL Multiphysics Environment. The use of COMSOL Multiphysics environment will have a great impact on such applications i.e. electrostatic liquid spraying, yet to be explored significantly [18]. So far, no statistical techniques are reported for flow processes in electrostatic liquid spraying $[19,20]$. Recently, a study has been published by Murtadha and his associates, showed the application of COMSOL Multiphysics in agricultural liquid spraying processes; however the manuscript lacks the fundamental technical details and appropriate specifications for the design of experiments [21] This paper didnot specify the flow rate of the nozzle, exact geometrical boundaries etc. A computational model has been developed using COMSOL to understand the effects of the electrode geometry, deposition target and spray cloud the induction charging parameters are optimized. The developed computational model is validated through the experimental results. The experimental results are in good agreement with the computational model of electrostatic charging of liquid sprays. 


\section{METHODOLOGY}

In electrostatic spraying, it is desirable to charge the particle as high as possible and the charged droplet must be acted upon by an electric field. A major portion of the electrostatic spraying research has been in the development of reliable means for finely divided particulate charging. The level of charge onto the droplet depends on charging time constant and the charging time constant is directly associated with liquid conductivity. The performance can be defined in terms of charge to mass ratio, which depends on applied high voltage, liquid conductivity, electrode material, electrode geometry, liquid flow rate, supplied air pressure, deposition target etc. Keeping this view point of optimizing the effects of electrode geometry of spray charging through induction charging, deposition target and spray cloud, a computational model is developed using COMSOL Multiphysics environment version 4.3 and experimental evaluation is carried out.

By using AC/DC module, concept of electrostatics has been implemented to analyze the resultant electric field. Finite Element Model (FEM) as shown in Fig. 1 consists of a cubical block $(1000 \times 1000 \times 1000 \mathrm{~mm})$ with boundary conditions, a deposition target of different shapes and a charged spherical droplet of $50 \mu \mathrm{m}$ Volume Median Diameter (VMD). The water droplet was charged to a significant level of surface charge density of $3.05 \times 10^{-5} \mathrm{Cm}^{-2}$ which was placed inside the block at $100 \times 500 \times 500 \mathrm{~mm}$ in $\mathrm{x}, \mathrm{y}$ and $\mathrm{z}$-axis respectively. All different shaped targets were of the same size having radius of $50 \mathrm{~mm}$ and height of $100 \mathrm{~mm}$, placed at $850 \times 500 \times 500 \mathrm{~mm}$ in $\mathrm{x}, \mathrm{y}$ and $\mathrm{z}$-axis respectively to align properly with the charged water droplet.

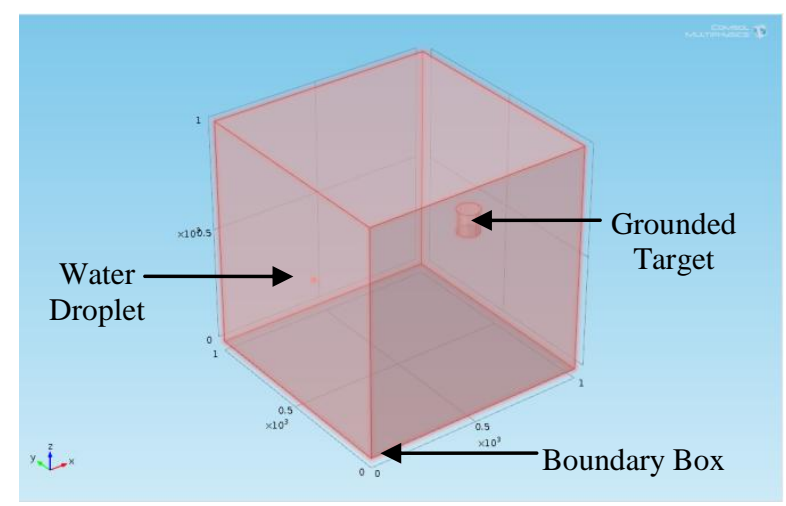

Fig 1: Computational model for calculation of electric field distribution of charged water droplet with respect to grounded target.

The material provided to cubical block, liquid droplet and deposition target was air, water and copper respectively. With the implication of extra fine meshing, results were computed to analyze the electric field norm. by applying the Poisson equation of electrostatics and COMSOL FEM model was solved. Poisson equation originated from Maxwell's equations with the definite boundary conditions.

$E=-\nabla \cdot V$

$\nabla .\left(\varepsilon_{0} \varepsilon_{r} E\right)=\rho_{s}$

Where, $E$ is electric field $\left(\mathrm{Vm}^{-1}\right), \mathrm{V}$ is electric potential $(\mathrm{V})$, $\nabla$ is Del operator, $\rho_{v}$ is the charge density of liquid droplet $\left(\mathrm{Cm}^{-2}\right), \quad \varepsilon_{0}$ is permittivity of free space $\left(8.854 \times 10^{-12} \mathrm{Fm}^{-1}\right)$ and $\varepsilon_{r}$ is relative permittivity.
The electric force per unit droplet mass $F_{E}$ the droplet can be expressed as

$F_{E}=E \cdot(q / m)_{p}$

Where $(q / m)_{p}$ is the charge to mass ratio of an individual droplet.

\section{EXPERIMENTS}

Laboratory experiments were carried out to observe and evaluate the electric field distribution of induction charging and deposition characteristics and hence charge to mass ratio of an air-assisted electrostatic nozzle designed and developed at CSIR-CSIO and all rights are reserved. The laboratory experiments were conducted in open air and at ambient conditions $(\mathrm{T}=288 \pm 2 \mathrm{~K}, \mathrm{RH}=50 \pm 3 \%)$. The experimental setup of an air-assisted electrostatic nozzle performance evaluation is shown in Fig. 2.

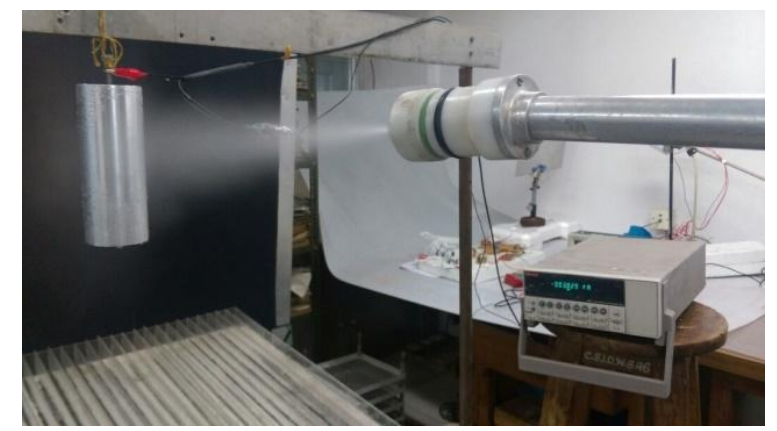

Fig 2: Experimental set-up of an air-assisted electrostatic nozzle for the evaluation of charge to mass ratio.

The liquid droplet was charged to a significant level of charge by applying high voltage to the induction electrode embedded in the nozzle using high voltage module (ULTAVOLT +20 $\mathrm{kV}, 1.5 \mathrm{~mA}, 30 \mathrm{~W}$, for laboratory experiments only). A range of high voltages $(0,0.25,0.5,0.75,1.0,1.25,1.5,1.75,2.0$, 2.25 and $2.5 \mathrm{kV}$ ) were applied to the electrostatic nozzle in the experiments. The liquid flow rate of the electrostatic nozzle was $110 \mathrm{mlm}^{-1}$ with a slight variation in flow as the pressure of air supply changed from 1-3 bars. The compressed air was provided from the air compressor (Model No: SAN10101, ELGI Equipment Ltd., CFM 3.89), air regulator for regulated air supply (Model No: LOE-D-MINI C643, FESTO) along with air flow meter (Model No: PFM711S-C8C-A-WS-X731). The spray current was measured with a programmable Electrometer (Model No.6514, Keithley) connected to the target.

\section{RESULTS AND DISCUSSION \\ 4.1. Effect of electrode geometry on electric field distribution}

In this case, electrodes were square electrode of square cross section, circular electrode of square cross section, square electrode of circular cross-section and circular electrode of circular cross section of 4.0 and $9.0 \mathrm{~mm}$ of inner and outer diameters respectively. The copper ( $98 \%$ pure) electrodes were placed at the distance of $2.5 \mathrm{~mm}$ from the nozzle tip, held at an optimized position from the liquid jet film with the support of holder made up of insulating material. The maximum electric field norm. of different geometry electrode is enamurated in Table 1. 
Table 1. Maximum electric field (norm.) of different geometry electrodes.

\begin{tabular}{l|lc}
\hline $\begin{array}{l}\text { S. } \\
\text { No. }\end{array}$ & Electrode Geometry & $\begin{array}{l}\text { Maximum Electric } \\
\text { Field norm. }(\text { V/m })\end{array}$ \\
\hline 1. & $\begin{array}{l}\text { Square electrode with } \\
\text { circular cross-section }\end{array}$ & $4.48 \times 10^{6}$ \\
2. & $\begin{array}{l}\text { Circular electrode with } \\
\text { circular cross-section }\end{array}$ & $2.20 \times 10^{6}$ \\
3. & $\begin{array}{l}\text { Circular electrode with } \\
\text { square cross-section } \\
\text { Square electrode with } \\
\text { square cross-section }\end{array}$ & $1.22 \times 10^{6}$ \\
\hline
\end{tabular}
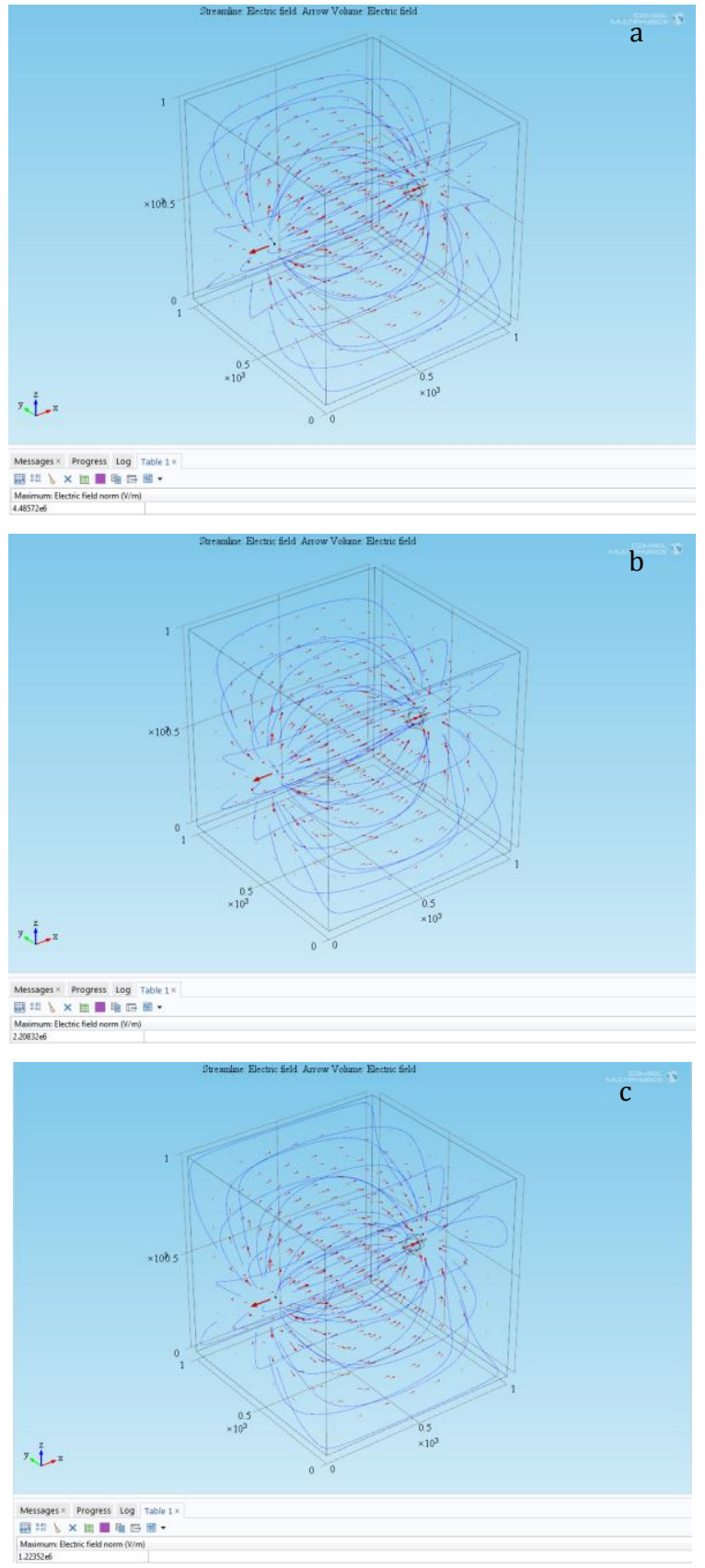

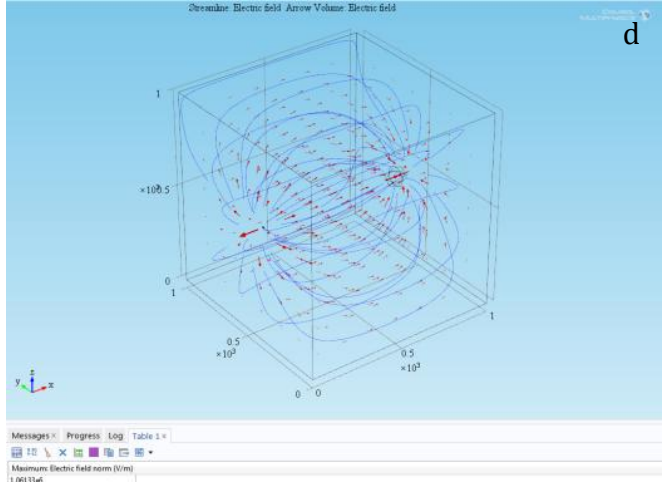

Fig 3: Effect of electrode geometry on electric field distribution (a) Square electrode with circular crosssection (b) Circular electrode with circular cross-section (c) Circular electrode with square cross-section (d) Square electrode with square cross-section.

The amount of induced charge may be expressed as $q_{t}=$ $\varepsilon_{0} \varepsilon_{r} \int E_{n} d A$. For the given construction of nozzle body which dictates the jet break-up process, this effect must be achieved by properly selecting the geometry of charging electrode arrangement. [22-24]. The plot of electric field lines and aerovolume distribution is shown in Fig. 3, which shows the maximum electric field distribution of different electrode geometry.

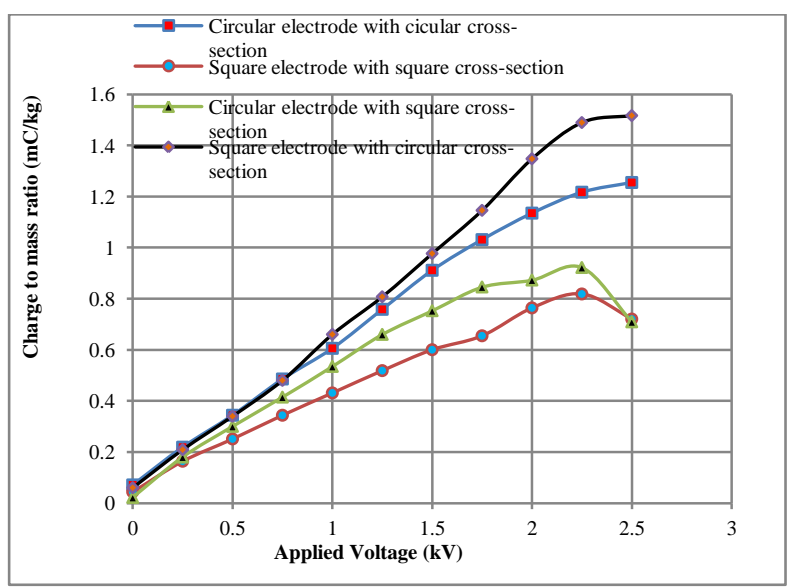

Fig 4: Charge to mass variation with the change in electrode geometries.

Fig. 4 showed that square electrode of circular cross section performed better in terms of charge-to-mass ratio as compared to the other electrode geometries. However, due to geometrical inconvenience, the ring electrode has been optimized and can be used for further study. One of the key finding of the experiment was square electrode, more efficient at charging due to the non-linearity of the electric field which induces more efficient discharging of ions to the continuum phase. It is also observed that at higher voltages, the charge to mass decreases in case of sharp edges electrode, because of corona discharge and breakdown failure.

\subsection{Effect of deposition target on electric field distribution}

In this case, the computational modeling was developed to observe the effect of deposition target in electrostatic spraying processes. The electric field distribution of the charged water droplet-target model for an ellipsoid, conical, spherical and cylindrical target is shown in the Fig. 5. 

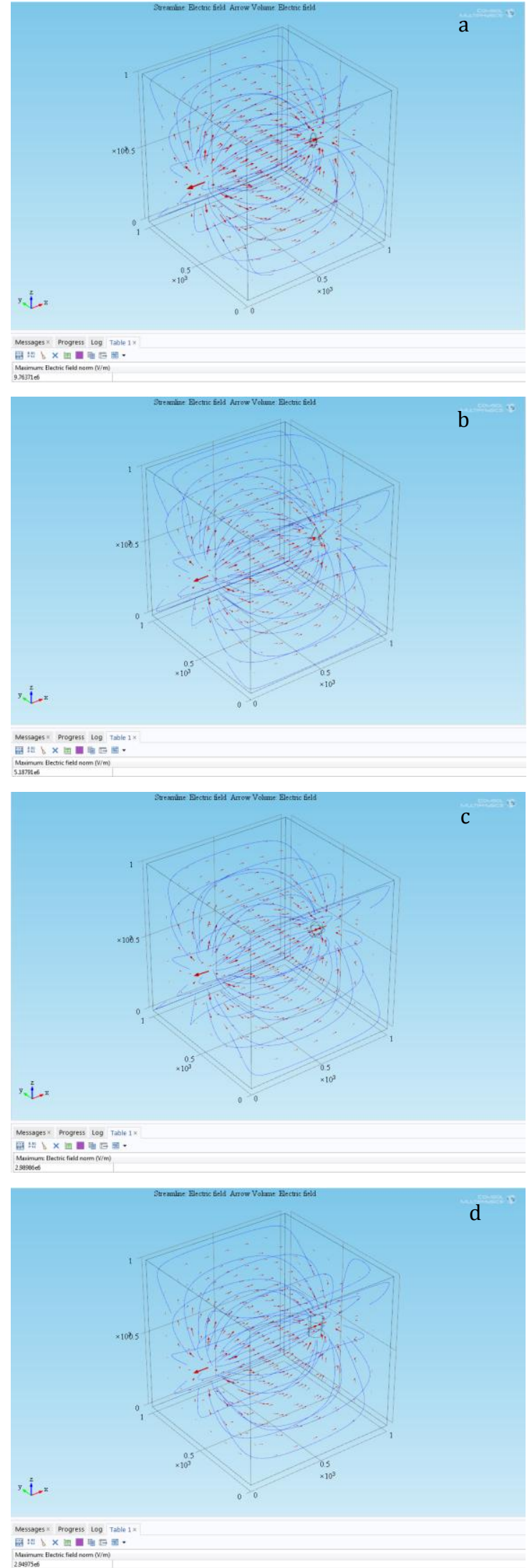

Fig 5: effect on electric field distribution of different shaped deposition target (a) Elliptical (b) Conical (c) Spherical (d) Cylindrical.

The maximum electric field achieved, for different deposition targets were summarized in the Table 2 .
Table 2. Maximum electric field (norm.) of charged liquid droplet with varying deposition target.

\begin{tabular}{c|lc}
\hline S. No. & $\begin{array}{l}\text { Shape of the } \\
\text { Target }\end{array}$ & $\begin{array}{l}\text { Maximum Electric } \\
\text { Field norm. }(\text { V/m })\end{array}$ \\
\hline 1. & Elliptical & $9.76 \times 10^{6}$ \\
2. & Conical & $5.18 \times 10^{6}$ \\
3. & Spherical & $2.98 \times 10^{6}$ \\
4. & Cylindrical & $2.94 \times 10^{6}$ \\
\hline
\end{tabular}

Elliptical shaped target generates maximum electric field (norm.) and a cylindrical target generates the least electric field. These results will be used in our developed air-assisted electrostatic sprayer so that amount of pesticide can be controlled on the basis of the shape and size of targets.

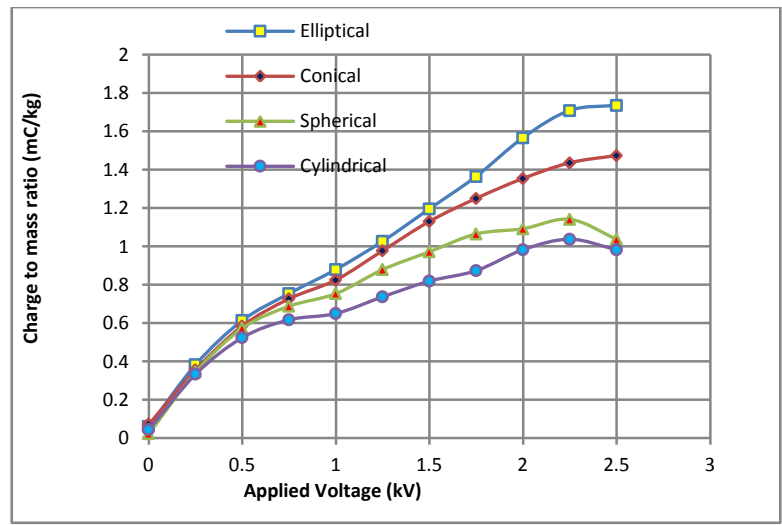

Fig 6: Charge to mass variation of electrostatic nozzle spraying with the variation of deposition target.

From the laboratory experiments as shown in Flig. 6, he relationships between the spray current $(\mu \mathrm{A})$ and hence charge to mass ratio and deposition target, water flow rate, applied voltage are explained.

\subsection{Effect of spray cloud on electric field distribution}

In this case, the cylindrical deposition target was given a negative potential varied from 0 to 2000 volts $(0,500,1000$, 2000 volts). Fig. 7 showed that the spray cloud expands with increased droplet charge-to-mass ratio due to increased space charge near the deposition target. This increases the spray uniformity and deposition but at the same time increases radial drift. The study of radial drift and deposition efficiency of electrostatic spraying are carried out through the experiments. In order to increase the deposition and reduce the droplet motion to the nearby ground, the droplet chargeto-mass ratio needs to be carefully selected.

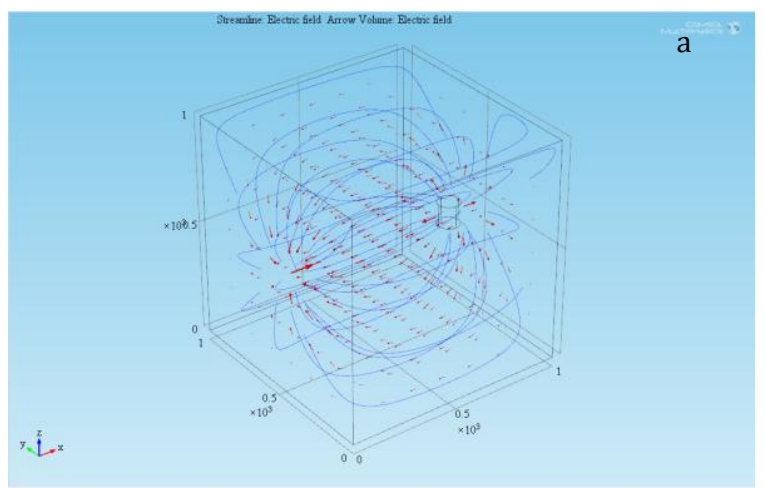



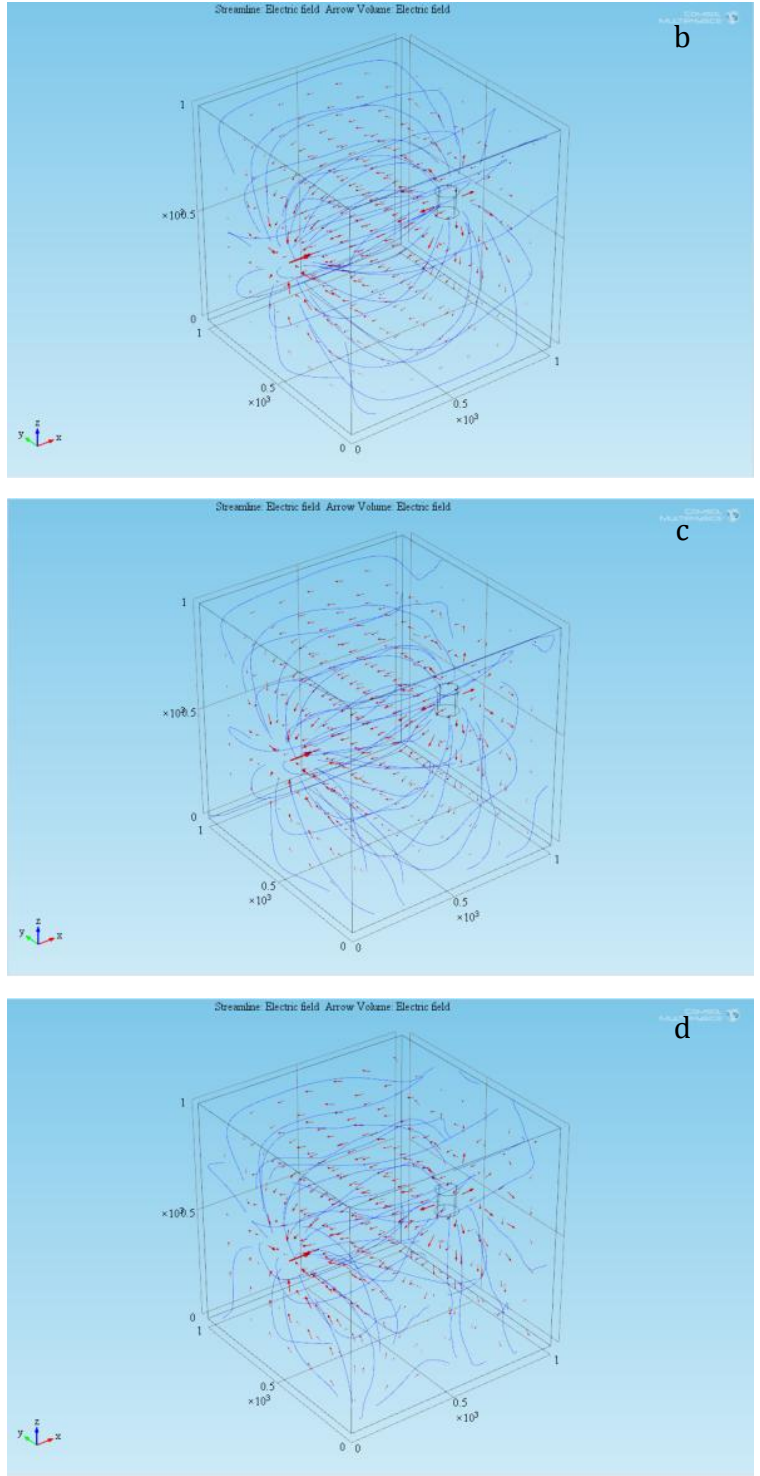

Fig 7: Effect of spray cloud near the deposition target onto the electric field distribution with the applied voltage to the target (a) $0 \mathrm{~V}$ (b) $-\mathbf{5 0 0} \mathrm{V}$ (c) $-1000 \mathrm{~V}$ and (d) $-2000 \mathrm{~V}$.

When there is no voltage on deposition target, the electric field lines sweep over the cylinder and some of them may enter at the front and up and the rest of pass by the target. When the cylindrical target supplied a negative voltage of 500 volts, the field lines were distorted in the nature, due to repulsive nature of negative spray cloud. Simultaneously, in case of different applied voltage of -1000 and -2000 volts, the electric field lines were almost trying to distort from the target to get grounded objects. As the target distance decreased, the volume of spray cloud decreased which has reduced the space charge repulsion and thus reduced the radial drift.

\section{CONCLUSION}

COMSOL FEM model was developed to optimize the electric field distribution and hence charge to mass ratio of four different geometry electrodes, four different grounded deposition targets and spray cloud of different levels for a fixed charged water droplet. Using the analogy of the Poisson equation to the scalar transport equations, the electric field can be solved within COMSOL Multiphysics, and the electrostatic spraying process can be modeled with various parameters and complex geometries. For the air-assisted electrostatic induction charging spraying process, the modeling results showed that the spray cloud expands with increased droplet charge-to-mass ratio due to increased space charge. This increases the spray uniformity and deposition but at the same time increases radial drift. They need to be independently controlled in order to increase the transfer efficiency and reduce drift. There is good conformity between the theoretical and practical results. The results obtained in this work will be used to design and develop an air-assisted electrostatic nozzle for agricultural applications.

\section{ACKNOWLEDGMENTS}

The Authors are thankful to the Department of Science and Technology (DST), New Delhi for the financial assistance. We thank to Director, CSIR-Central Scientific Instruments Organisation, Chandigarh for creating the healthy work environment to carry out the Project. We are also thankful to CSIR-CMERI-CoEFM, Ludhiana and Punjab Agriculture University (PAU), Ludhiana to provide the platform for the field trials.

\section{REFERENCES}

[1] M.K. Patel, Hemant K Sahoo, Manoj K Nayak, Ashwani Kumar, C Ghanshyam, and Amod Kumar, "Electrostatic Nozzle: New Trends in Agricultural Pesticides Spraying”, SSRG International Journal of Electrical and Electronics Engineering, 2015, pp. 6-11.

[2] M.K. Patel, C. Ghanshyam, and P. Kapur, "Characterization of electrode material for electrostatic spray charging: Theoretical and Engineering Practices", Journal of Electrostatics, vol. 71, 2013, pp. 55-60.

[3] V.R. Mamidi, C. Ghanshyam, M.K. Patel, and P. Kapur, "Electrostatic hand pressure Knapsack spray system with enhanced performance for small scale forms", Journal of Electrostatics, vol. 71, no. 4, 2013, pp. 785-790.

[4] Y. Ru, J.Q. Zheng and H.P. Zhou, "Design and test study on double acicular electrostatic device of High-range Electrostatic Sprayer", Journal of Nanjing Forestry University, vol. 31 , no. 6, 2007, pp. 87-90.

[5] G.N. Laryea, and S.Y. No, "Development of electrostatic pressure-swirl nozzle for agricultural applications", Journal of Electrostatics, vol. 57, no. 2, 2003, pp. 129142.

[6] N. Chigier, "Challenges for future research in atomization and spray technology: Arthur Lefebvre Memorial Lecture", 16, 2006, pp. 727-736.

[7] Y. Ru, J.Q. Zheng, H.P. Zhou, and C. Shu, "Electric field distribution produced by circular electrode of induce charging nozzle", Transactions of Chinese Society of Agriculture and Engineering. 5, 2008.

[8] B.M. Maynagh, B. Ghobadian, and M.R. Jahannama, "Effect of electrostatic induction parameters on droplets charging for agricultural application", Journal of Agricultural Science and Technology, vol. 11, no. 3, 2009, pp. 249-257.

[9] S. Zhao, G.S.P. Castle and K. Admiak, "The effect of space charge on the performance of an electrostatic induction charging spray nozzle", Journal of Electrostatics, vol. 63, no. 3-4, 2005, pp. 261-272. 
[10] R.S. Sasaki, M.M. Teixeira, H.C. Fernandes, P. M. de B. Monteiro, D.E. Rodrigues, and C. B. de Alvarenga, "Parameters of electrostatic spraying and its influence on the application efficiency", Revsta Ceres, vol. 60, no. 4, 2013, pp. 474-479.

[11] S. Zhao, G.S.P. Castle, and K. Adamiak K., "Comparison of conduction and induction charging in liquid spraying", Journal of Electrostatics, vol. 63, no. 610, 2005, pp. 871-876.

[12] M.K. Patel, C. Ghanshyam, V.R Mamidi, and Pawan Kapur, "Selection of electrode material for spray charging in electrostatic nozzle", The Journal of Instrument Society of India, vol. 42, no. 4, 2012, pp. 272-275.

[13] V.R. Mamidi, C. Ghanshyam, M.K. Patel, and Pawan Kapur, "Electrostatic Hand Pressure Swirl Nozzle for Small Crop Growers", International Journal of Applied Science and Technology Research Excellence, vol. 2, no. 2, 2012, pp. 164-168.

[14] W. Machowski, and W. Balachandran, "Design of Electrostatic Fog Generator Using a Reverse Field Modeling Technique", Industry Applications Conference, Thirty-Second IAS Annual Meeting, IAS, vol. 97, no. 3, 1997, pp. 1784-1789.

[15] D. Maski, and D. Durairaj, "Effects of electrode voltage, liquid flow rate, and liquid properties on spray chargeability of an air-assisted electrostatic-induction spray-charging system", Journal of Electrostatics, vol. 68,2010 , pp. 152-158

[16] A. Chaim, M.C.P.Y. Pessoa, and V.L. Ferracini, "Eficiência de deposição de pulverização em videira comparando bicos e pulverizadores" Pesticidas: revista de ecotoxicologia e meio ambiente, vol. 14, 2004, pp. $39-46$.

[17] J.S. Shrimpton, "Electrohydrodynamics of charge injection atomization: regimes and fundamental limits", Atomization and Sprays, vol. 13, 2003, pp. 173-190.
[18] M.K. Patel, C. Ghanshyam, V.R. Mamidi, and Pawan Kapur, "Performance and Characterization of Different Material Electrodes in Electrostatic Pesticide Spraying Nozzle System", International Journal of Applied Science and Technology Research Excellence, vol. 2, no. 2, 2012, pp. 158-163.

[19] Y. Ru, H. Zhou and J. Zheng, "Design and Experiments on Droplet Charging Device for High Range Electrostatic Sprayer" Book- Pesticides in the Modern World- Pesticides Use and Management, 2011.

[20] W. Jia, F. Xeu, B. Qui and Z. Wang "Design and Performance of Inductive Electrostatic Sprayer", Research Journal of Applied Sciences, Engineering and Technology, vol. 5, no. 21, 2013, pp. 5102-5106.

[21] M. Al-Mamury, N. Manivannan, H. Al-raweshidy, and W. Balachandran, "Computation Model of Electrostatic Spraying in Agriculture Industry", Excerpt from the Proceedings of the 2014 COMSOL Conference in Cambridge, 2014.

[22] W. Balachandran, D. Hu, A. Yule, J.S. Shrimpton, and A.P. Watkins, "Electro-statically produced fuel sprays for combustion applications", Fuel and Energy Abstracts, vol. 38 , no. 6,1997 , pp. 421-422.

[23] M.K. Patel and C. Ghanshyam, "Fundamentals of Electrostatic Spraying: Basic Concepts and Engineering Practices", in: Srivastava, K., Singh, S. (Eds.), Uncovering New Methods for Ecosystem Management through Bioremediation, IGI Global Publishing, 2015, pp. 468-495.

[24] E.A. Badea, J. Oesterheld, S. Friedel and M. Olsson, "Accessing COMSOL Performances on Typical Electromagnetic Problems Faced by Turbo-Generator Manufacturers" Excerpt from the Proceedings of the 2012 COMSOL Conference in Milan, 2012. 